\title{
Transcellular Water Flow Modulates Water Channel Exocytosis and Endocytosis in Kidney Collecting Tubule
}

\author{
Michio Kuwahara, Lan-Bo Shi, F. Marumo, ${ }^{\star}$ and A. S. Verkman \\ Departments of Medicine and Physiology, Cardiovascular Research Institute, University of California, San Francisco, California 94143; \\ and *Department of Internal Medicine, Tokyo Medical and Dental University, Tokyo, Japan
}

\begin{abstract}
The regulation of osmotic water permeability $\left(P_{\mathbf{f}}\right)$ by vasopres$\sin (V P)$ in kidney collecting tubule involves the exocytic-endocytic trafficking of vesicles containing water channels between an intracellular compartment and apical plasma membrane. To examine effects of transcellular water flow on vesicle movement, $P_{f}$ was measured with 1-s time resolution in the isolated perfused rabbit cortical collecting tubule in response to addition and removal of $\mathrm{VP}(250 \mu \mathrm{U} / \mathrm{ml})$ in the presence of bath $>$ lumen $(B>L)$, lumen $>$ bath $(L>B)$, and lumen $=$ bath $(L=B)$ osmolalities. With VP addition, $P_{f}$ increased from 12 to $240-$ $270 \times 10^{-4} \mathrm{~cm} / \mathrm{s}\left(37^{\circ} \mathrm{C}\right)$ in $10 \mathrm{~min}$. At $1 \mathrm{~min}, P_{\mathrm{f}}$ was $\sim 70 \times$ $10^{-4} \mathrm{~cm} / \mathrm{s}$ for $B>L, L>B$, and $L=B$ conditions. At later times, $P_{f}$ increased fastest for $L>B$ and slowest for $B>L$ osmolalities; at $5 \mathrm{~min}, P_{\mathrm{f}}$ was $250 \times 10^{-4} \mathrm{~cm} / \mathrm{s}(\mathrm{L}>B)$ and 158 $\times 10^{-4} \mathrm{~cm} / \mathrm{s}(B>L)$. With VP removal, $P_{f}$ returned to pre-VP levels at the fastest rate for $B>L$ and the slowest rate for $L>B$ osmolalities; at $30 \mathrm{~min}, P_{f}$ was $65 \times 10^{-4} \mathrm{~cm} / \mathrm{s}(B>L)$ and 183 $\times 10^{-4} \mathrm{~cm} / \mathrm{s}(\mathrm{L}>\mathrm{B})$. For a series of osmotic gradients of different magnitudes and directions, the rates of $P_{f}$ increase and decrease were dependent upon the magnitude of transcellular volume flow; control studies showed that paracellular water flux, asymmetric transcellular water pathways, or changes in cell volume could not account for the data. VP-dependent endocytosis was measured by apical uptake of rhodamine-dextran; in paired studies where the same tubule was used for + and - gradients, $B>L$ and $L>B$ osmolalities gave $168 \%$ and $82 \%$ of uptake measured with no gradient. In contrast, endocytosis in proximal tubule was not dependent on gradient direction. These data provide evidence that transcellular volume flow modulates the vasopressin-dependent cycling of vesicles containing water channels, suggesting a novel driving mechanism to aid or oppose the targeted, hormonally directed movement of subcellular membranes. (J. Clin. Invest. 1991. 88:423-429.) Key words: endocytosis - kidney collecting tubule - vasopressin • water permeability
\end{abstract}

Address correspondence to Dr. Alan S. Verkman, 1065 Health Sciences East Tower, Cardiovascular Research Institute, University of California, San Francisco, CA 94143.

Received for publication 28 November 1988 and in revised form 26 March 1991.

J. Clin. Invest.

(C) The American Society for Clinical Investigation, Inc. 0021-9738/91/08/0423/07 \$2.00

Volume 88, August 1991, 423-429

\section{Introduction}

The regulation of water permeability in vasopressin (VP) ${ }^{1}$-sensitive epithelia is thought to involve a cycle of exocytosis and endocytosis where intracellular vesicles containing water channels are inserted into and removed from the cellular apical membrane $(1,2)$. There is a good correlation between the presence of apical membrane particle aggregates and transepithelial osmotic water permeability in toad bladder granular cells and kidney collecting tubule cells involved in the hydroosmotic response to vasopressin (3-5). Endosomes isolated from toad urinary bladder that were formed in response to vasopressin contained functional water channels that might be recycled between subcellular and plasma membrane compartments (6). Endosomes containing the vasopressin-sensitive water channel had low urea and proton permeabilities (7), and very high water permeability that was not subject to direct biochemical regulation (8). The signaling mechanism responsible for the modulation of membrane cycling is believed to involve a cAMP-dependent protein kinase and the cell cytoskeleton (9); however, the biochemical and physical factors controlling exocytosis and endocytosis have not been established.

In kidney collecting tubule, endocytosis of water channels probably occurs by formation of clathrin-coated pits (10-12); however, little is known about the fate of endocytosed membranes or of the mechanism by which intracellular membranes fuse with the apical surface in response to vasopressin addition. Endosomes formed in the papillary collecting tubule from Brattleboro rats contained functional water channels only when the rats had received vasopressin or the $V_{2}$ agonist DDAVP before endosome isolation $(13,14)$, providing strong evidence for a water channel shuttle mechanism in kidney collecting tubule. The vasopressin-induced endosomes did not acidify in response to ATP, and endocytosed fluid phase markers did not colocalize with lysosomes, raising the interesting possibility that intracellular processing of endosomes containing the vasopressin-sensitive water channel does not involve the conventional endocytotic pathway of progressive acidification (15). In kidney proximal tubule, there is rapid constitutive (hormone-independent) endocytosis that also involves clathrin-coated vesicles $(12,16,17)$. However, in contrast to collecting tubule, endosomes from apical membrane of proximal tubule contain functional water channels and do fuse

1. Abbreviations used in this paper: CCT, cortical collecting tubule; FS, fluorescein sulfonate; $J_{v}$, transepithelial water flow; $P_{f}$, osmotic water permeability; PST, proximal straight tubule; $\left(T_{1 / 2}\right)_{o n}$, half-time for turnon phase of $P_{f} ;\left(T_{1 / 2}\right)_{\text {off }}$, half-time for turn-off phase of $P_{f} ; V P$, vasopres$\sin$. 
with acidic intracellular vesicles (18); endosome acidification may be regulated by protein kinase A-dependent phosphorylation of a chloride channel (19).

The interesting observation has been made in a number of laboratories that the presence of a serosal-to-mucosal osmotic gradient (serosal $>$ mucosal osmolality) appears to alter the endocytic phase of membrane cycling in toad urinary bladder (20-22). A serosal-to-mucosal osmotic gradient both increases the endocytic retrieval of fluid-phase fluorescent markers, and enhances the downregulation of water permeability in response to prolonged vasopressin stimulation. There are no data available to establish a mechanism for this phenomenon. It is not known whether similar phenomena occur in kidney collecting tubule. In addition, there have been no studies of the effect of osmotic gradients on membrane exocytosis, or of the effects of oppositely directed gradients on exocytosis or endocytosis.

We have used a new fluorescence method to test the hypothesis that transcellular water flow modulates exocytosis and endocytosis of the vasopressin-sensitive water channel in kidney collecting tubule. Experiments were performed by in vitro perfusion of cortical collecting tubule from rabbit. The collecting tubule is a unique system to examine the flow hypothesis because transcellular volume flow is rapid, hormone sensitive, not unstirred layer limited, and can be reversed in direction. The time course of osmotic water permeability in response to changes in bath osmolality and to addition and removal of vasopressin was measured from the fluorescence of an impermeant fluorophore perfused through the tubule lumen $(23,24)$. Unlike studies in toad urinary bladder, tubule osmotic water transport measurements are not subject to significant unstirred layer effects that restrict the binding and unbinding of vasopressin, and cause solute polarization within the unstirred layer (25).

We present evidence that rates of water channel insertion and retrieval in kidney collecting tubule are influenced by the magnitude and direction of water flow passing through the cell; water movement across the apical membrane from luminal solution to cell interior decreases exocytosis and increases endocytosis, whereas water movement from cell interior to luminal solution increases exocytosis and decreases endocytosis. Comparative studies in proximal and collecting tubule of the influence of osmotic gradient direction on the endocytosis of fluorescent markers showed that the modulation of endocytosis by transcellular water flow is selective for vasopressin-sensitive trafficking of water channels in collecting tubule. A series of control experiments were performed to rule out effects of absolute bath osmolality, asymmetric routes for VP-independent and dependent water flow and changes in cell volume. Our results suggest that in kidney collecting tubule, both hormonal and physical factors modulate the targeted movement of intracellular membranes. Because interstitial osmolality in renal cortex is always $\sim 300$ mosM, our results suggest that the dynamic control of water absorption by vasopressin is influenced by the osmolality of the tubular fluid.

\section{Methods}

In vitro microperfusion system. Isolated segments of rabbit cortical collecting tubule (CCT) or proximal straight tubule (PST) were dissected and perfused in vitro as described previously $(23,26)$. Kidneys from New Zealand white rabbits $(1.5-2.5 \mathrm{~kg})$ were cut in coronal slices. Tubules were dissected in a cooled $\left(4^{\circ} \mathrm{C}\right)$ bath solution and transferred to a bath of $200 \mu \mathrm{l}$ vol. Tubules (length $1.0-1.8 \mathrm{~mm}$ ) were mounted between holding pipettes and the lumen was cannulated for perfusion. Luminal perfusion rate was maintained at $10-15 \mathrm{nl} / \mathrm{min}$ with a nanoliter infusion pump (Harvard Apparatus, Inc., S. Natick, MA) driving a 10- $\mu$ l Hamilton syringe which was connected to the perfusion pipette with polyethylene tubing. Luminal perfusion rate was calibrated against set pump rates as described previously (27).

The bath solution was preheated to $37^{\circ} \mathrm{C}$ and was exchanged continuously at $10-20 \mathrm{ml} / \mathrm{min}$. Bath fluid composition was changed by adjusting a five-way valve near the bath. The isosmotic bath solution contained (in mM): $142 \mathrm{NaCl}, 5 \mathrm{KCl}, 5$ glucose, $1 \mathrm{CaCl}_{2}, \mathrm{pH}$ 7.4. The luminal perfusate contained in addition $1 \mathrm{mM}$ fluorescein sulfonate (FS; Molecular Probes, Inc., Junction City, OR) which has been shown to be an impermeant luminal fluorophore. For studies in which lumen and bath osmolalities were different, the composition of the solution with lower osmolality was that of the isotonic solution in which the appropriate amount of $\mathrm{NaCl}$ was removed; sucrose was added to this solution to prepare the solution of higher osmolality. Experiments in PST were performed using 40 mosM osmotic gradients (lumen 290, and bath 250,290 , or 330 mosM). Solution osmolalities were measured with a vapor pressure osmometer (Wescor Inc., Logan, UT) and were adjusted to desired values by addition of $\mathrm{NaCl}$ or $\mathrm{H}_{2} \mathrm{O}$. For vasopressin experiments, synthetic arginine vasopressin (Pitressin; Parke-Davis, Morris Plains, NJ) was added to the bath solution at a concentration of $250 \mu \mathrm{U} / \mathrm{ml}$.

Fluorescence measurement system. Luminal FS fluorescence was measured using an inverted epifluorescence microscope (Nikon Diaphot). Tubules were viewed through a $\times 25$ long working distance objective (numerical aperture 0.35; Leitz Wetzlar). Fluorescence was excited using a 100-W tungsten lamp powered by a stabilized DC supply (Oriel Corp., Stratford, CT) in series with a 1.0 neutral density filter and a KG-3 infrared blocking filter (Schott Optical Glass Inc., Duryea, PA).

For measurement of luminal FS fluorescence, excitation was at $480 \pm 5 \mathrm{~nm}$, with 510 -nm dichroic mirror and $>530$-nm emission cuton filter. Fluorescence was detected by a photomultiplier (R928S; Hamamatsu Corp., Middlesex, NJ) contained in a cooled housing (FACT 50; Thorn EMI Gencom, Inc., Plainview, NY). The signal was amplified by a DC power supply and amplifier (Ealing Corp., S. Natick, MA) and interfaced to an IBM PC/AT computer via an ADALAB-PC analogue-to-digital interface board (Interactive Microware, State College, PA). The signal was filtered electronically using a single pole RC filter with 0.3-s time constant; data were acquired at a rate of 30 points/s and averaged over 1-s intervals. In some experiments luminal FS fluorescence was measured by a SIT camera using an ARGUS-100 imaging system (Hamamatsu Photonics).

Water permeability measurements. To eliminate effects of endogenous vasopressin, tubules were perfused for $90 \mathrm{~min}$ with vasopressinfree solution at $37^{\circ} \mathrm{C}(23,27)$ at a lumen flow rate of $10 \mathrm{nl} / \mathrm{min}$ and bath exchange rate of $5 \mathrm{ml} / \mathrm{min}$. For measurement of osmotic water permeability $\left(P_{f}\right)$, FS fluorescence was monitored at the tip of the holding pipette (inner diameter $\sim 40 \mu \mathrm{m}$ ) just distal to the end of the tubule segment. As described previously (23), the advantages of this method are lack of effect on fluorescence signal of tubule motion and changes in tubule inner diameter, and elimination of photodynamic cell injury. The excitation and emission path contained iris diaphragms so that only the specified area was illuminated and measured. Under these conditions, background fluorescence was $<0.5 \%$ of total signal.

$P_{f}$ was calculated from the relation (28),

$P_{\mathrm{f}}=-\frac{V_{\mathrm{o}} C_{\mathrm{o}}}{A V_{\mathrm{w}}}\left[\frac{C_{\mathrm{o}}-C_{\mathrm{L}}}{C_{\mathrm{o}} C_{\mathrm{b}} C_{\mathrm{L}}}+\frac{1}{\left(C_{\mathrm{b}}\right)^{2}} \ln \frac{\left(C_{\mathrm{L}}-C_{\mathrm{b}}\right) C_{\mathrm{o}}}{\left(C_{\mathrm{o}}-C_{\mathrm{b}}\right) C_{\mathrm{L}}}\right]$

where $V_{o}$ is the initial lumen perfusion rate $\left(\mathrm{cm}^{3} / \mathrm{min}\right)$, $A$ is the inner tubule surface area $\left(\mathrm{cm}^{2}\right), V_{w}$ is the partial molar volume of water $(18$ $\left.\mathrm{cm}^{3} / \mathrm{mol}\right), C_{o}$ and $C_{L}$ are the initial and collected osmolalities, respectively, and $C_{b}$ is the bath osmolality. Because the fluorescence intensity of FS is linearly proportional to its concentration at $[\mathrm{FS}]<3 \mathrm{mM}$ (no self-quenching), $C_{L}$ was determined from the product of $C_{o}$ and the 
ratio of fluorescence intensities in the presence and absence of a transepithelial osmotic gradient. For examination of the relationship between driving force and water flow, transepithelial water flow $\left(\mathrm{J}_{\mathrm{v}} ; \mathrm{nl} / \mathrm{mm}\right.$ per min) was calculated from the relation $\mathrm{J}_{\mathrm{v}}=\left(V_{\mathrm{o}} / \mathrm{L}\right)\left(C_{\mathrm{\nu}} / C_{\mathrm{o}}-1\right)$, where $\mathrm{L}$ is tubule length $(\mathrm{mm})$. The log mean osmotic gradient was used to estimate the average driving force (29).

Endocytosis measurement. As above, tubules were first perfused and bathed in $290 \mathrm{mosM}$ solution for $90 \mathrm{~min}$ at $37^{\circ} \mathrm{C}$ in the absence of vasopressin. In CCT studies, vasopressin $(250 \mu \mathrm{U} / \mathrm{ml})$ was then added to the bath for $10 \mathrm{~min}$. At the time of vasopressin removal, rhodaminedextran $(25 \mathrm{mg} / \mathrm{ml}, 10 \mathrm{kD}$, neutral charge, Molecular Probes) was added to the luminal perfusate for uptake by apical endocytosis. The rhodamine-dextran was dialyzed overnight against $>500$ vol buffer to remove all unconjugated rhodamine as confirmed by TLC. After a 10-min labeling time, rhodamine-dextran was washed out and endocytosis was measured by the integrated rhodamine fluorescence intensity over a $0.1-\mathrm{mm}$ length of tubule. After $30 \mathrm{~min}$, bath osmolality was changed to 410 or $200 \mathrm{mosM}$ and vasopressin was added. After $10 \mathrm{~min}$, vasopressin was removed and rhodamine-dextran was added to the luminal perfusate as above. In this way the same tubule was used to measure endocytosis in the presence and absence of a transepithelial osmotic gradient. In control studies, rhodamine-dextran uptake was determined at a low temperature in which endocytosis was inhibited (see Results). In PST studies, rhodamine-dextran uptake was measured after 10-min labeling periods in the absence and then in the presence of an osmotic gradient.

Cell volume measurement. The methods described by Kirk et al. (30) and Strange and Spring (31) were used to measure the time course of principal cell volume in response to vasopressin addition and removal in the presence of bath $>$ lumen and lumen $>$ bath osmolalities Lumen flow was $10 \mathrm{nl} / \mathrm{min}$. Cells were imaged by quantitative light microscopy using a $\times 100$ oil immersion objective (numerical aperture 1.3). Cell volume was determined from lateral images of principal cells by manual cell tracing and computerized area integration. Principal cells were identified morphologically by established criteria including higher contrast, intracellular content, and rectangular border. Data were expressed as relative cell volume, where unity represented the volume before addition of an osmotic gradient. In some cells there was visible formation of "ADH-induced vacuoles" (32); the volume of all imaged principle cells was measured, including those with vacuoles.

\section{Results}

The time course of $P_{f}$ was measured in response to the addition and removal of bath vasopressin in the presence of bath $>$ lumen, lumen $>$ bath, and bath = lumen osmolalities (Fig. 1). After dissection, tubules were perfused and bathed in a 290mosM solution for 90 min to eliminate effects of endogenous vasopressin. For measurement of $\mathrm{P}_{\mathrm{f}}$ in response to a bath-to-lumen osmotic gradient (bath $>$ lumen osmolality) (trace $1 \mathrm{~A}$ ), bath osmolality was increased to $410 \mathrm{mosM}$, giving a low value for $P_{f}$ in the unstimulated tubule $\left(12 \pm 3 \times 10^{-4} \mathrm{~cm} / \mathrm{s}\right.$, mean \pm SEM, $n=10$ ). Upon addition of bath vasopressin, there was a lag period $(19 \pm 2 \mathrm{~s}, n=10)$ in which $\mathrm{P}_{\mathrm{f}}$ did not increase measurably, followed by an increase in $P_{\mathrm{f}}$ to a maximum value of $262 \pm 13 \times 10^{-4} \mathrm{~cm} / \mathrm{s}$ with a half-time of 4-5 min.

In response to vasopressin removal from the bath (trace 1 $B), P_{f}$ decreased to its baseline value with a half-time of $\sim 15$ min. Similar studies performed in the continuous presence of a lumen-to-bath osmotic gradient showed a similar lag time $(18 \pm 2 \mathrm{~s}, n=6)$ but a remarkably more rapid increase in $P_{\mathrm{f}}$ after vasopressin addition (trace $1 C$ ), and a slower decrease in $P_{\mathrm{f}}$ after vasopressin removal (trace $1 D$ ). To measure the time course of $P_{f}$ increase in the absence of an osmotic gradient, it

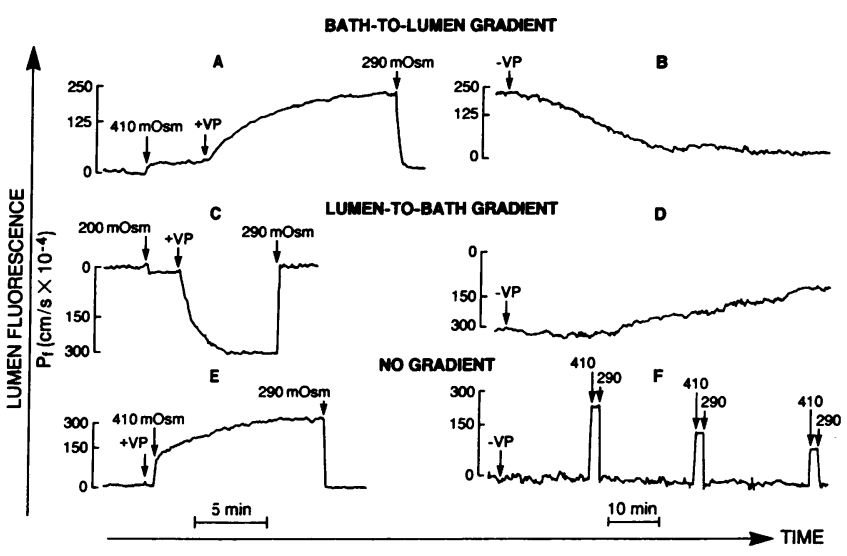

Figure 1. Time course of transepithelial osmotic water permeability in the isolated perfused cortical collecting tubule from New Zealand white rabbits. Luminal perfusion rate was $10 \mathrm{nl} / \mathrm{min}$; bath fluid was exchanged at $10-20 \mathrm{ml} / \mathrm{min}$. All measurements were performed at $37^{\circ} \mathrm{C}$. Where indicated, bath osmolality was changed and $250 \mu \mathrm{U} / \mathrm{ml}$ vasopressin was added to the bath solution. $P_{\mathrm{f}}$ values given on the ordinate were calculated from fluorescence intensities, tubule geometry, and lumen flow as described in Methods. $(A)$ and $(B)$ Bath-tolumen gradient. The time course of $P_{f}$ rise after vasopressin addition, and fall after vasopressin removal, are shown for a continuous 120 mosM bath-to-lumen gradient (bath 410 mosM, lumen 290 mosM). In trace $A$, the fluorescence decreased briskly after return of bath osmolality to $290 \operatorname{mosM}$ at the completion of the experiment. $(C)$ and (D) Lumen-to-bath gradient (bath 200 mosM, lumen 290 mosM). The time course of $P_{f}$ rise and fall for a continuous 90 mosM lumento-bath gradient. $(E)$ and $(F)$ No gradient. Tubules were bathed in a solution containing vasopressin for $45 \mathrm{~s}$ before imposing a 120 mosM bath-to-lumen gradient (curve $E$ ) The initial value of $\mathrm{P}_{\mathrm{f}}$ after addition of the gradient represents the rise in $P_{f}$ which occurred in the absence of a gradient. After vasopressin removal in the absence of a gradient (curve $F$ ), a measuring gradient was added briefly at 20 , 40 , and 60 min to quantitate the fall in $P_{f}$ which occurred in the absence of a gradient.

was necessary to measure $P_{f}$ by imposing an osmotic gradient at varying times after tubules were incubated with vasopressin in the absence of a gradient (trace $1 E$ ); $\mathrm{P}_{\mathrm{f}}$ was quantitated from the initial, immediate rise in fluorescence after addition of the gradient. Similarly, to measure the time course of $P_{f}$ decrease in the absence of an osmotic gradient, the "measuring" osmotic gradient was imposed briefly at varying times after vasopressin removal (trace $1 F)$.

The time courses of $P_{f}$ in response to addition and removal of vasopressin in a series of tubules are summarized in Fig. 2. In comparison to results obtained in the absence of an osmotic gradient, the increase in $P_{f}$ in response to vasopressin addition is enhanced by a lumen-to-bath osmotic gradient and inhibited by a bath-to-lumen osmotic gradient. In contrast, the decrease in $P_{f}$ in response to vasopressin removal is enhanced by a bathto-lumen gradient and inhibited by a lumen-to-bath gradient. Similar results were obtained by addition and removal of 8bromo-cAMP $(0.1 \mathrm{mM})$ in place of vasopressin, showing that the vasopressin-receptor interaction is not the site at which the osmotic gradient influences the turn-on and turn-off kinetics of $P_{\mathrm{f}}$. The direction of the measuring osmotic gradient did not affect results for the "no gradient" experiments, consistent with the observed symmetry in water transport (see below). Of note, the osmotic gradient had little influence on the rise in $P_{f}$ 


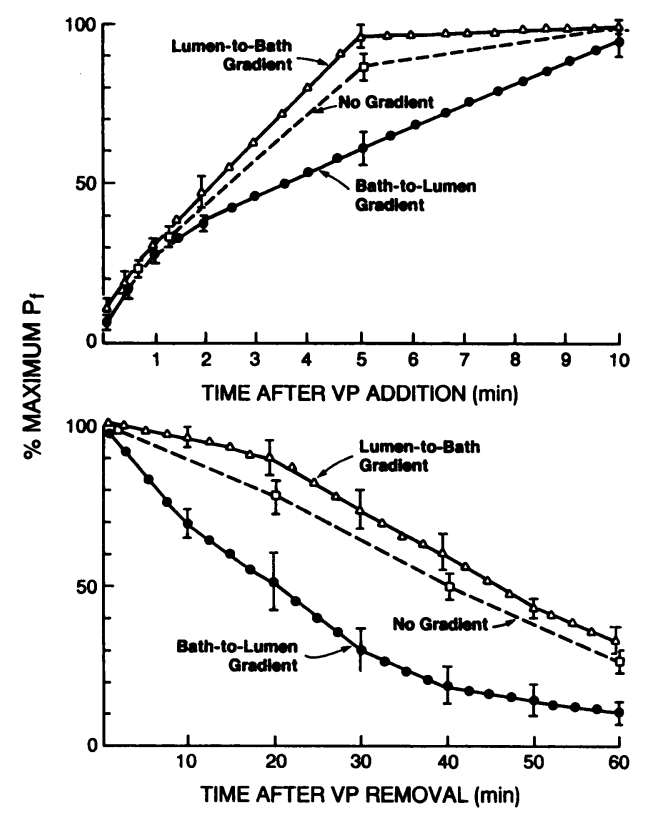

Figure 2. Dependence of the rise in $P_{f}$ after vasopressin addition, and the fall in $P_{\mathrm{f}}$ after vasopressin removal, on the presence and direction of a transepithelial osmotic gradient. Data (mean \pm SEM) obtained using the three protocols in Fig. 1 are summarized for 5-10 separate experiments. Data in the presence of bath-to-lumen and lumen-tobath gradients were obtained using continuous time courses for $P_{f}$ with $1 \mathrm{~s}$ time resolution (e.g., traces $1 A-1 D$ ); for clarity, data points were plotted every $0.5 \mathrm{~min}\left(\mathrm{P}_{\mathrm{f}}\right.$ increase) or every $2.5 \mathrm{~min}\left(\mathrm{P}_{\mathrm{f}}\right.$ decrease), with error bars given for some of the points. For vasopressin addition experiments, zero time was taken to be the time at which $P_{f}$ increased measurably after an initial lag period (range 16-23 s). The lag time did not differ significantly for bath-to-lumen and lumen-to-bath gradients (see text) and represents the pre-steady-state kinetics of signaling processes proximal to the step of water channel exocytosis (24).

over the first 2 min after vasopressin addition, at which time $P_{f}$ increased approximately fivefold over its baseline value. Further experiments were performed to support the interpretation of these results that it is transcellular water flow that modulates the kinetics of water channel exocytosis and endocytosis.

Experiments were performed for a series of different bath and lumen osmolalities to show that the turn-on and turn-off of water permeability is influenced by the magnitude and direction of transcellular water flow, rather than the absolute bath or lumen osmolalities. Fig. 3 shows that the size and direction of the osmotic gradient across collecting tubule epithelium does not influence significantly steady-state $P_{f}$ or the initial rate of turn-on of $\mathbf{P}_{\mathbf{f}}$ when transcellular water flow is relatively low. However, increasing osmotic gradients that induce lumen-tobath volume flow increase the half-time for the turn-on phase of $P_{f}$, and decrease the half-time for the turn-off phase of $P_{f}$. Similarly, gradients that induce bath-to-lumen volume flow decrease the half-time for turn-on $\left(T_{1 / 2}\right)_{\text {on }}$ and increase the halftime for turn-off of $P_{f}\left(T_{1 / 2}\right)_{\text {off }}$. Importantly, the kinetics of turn-on and turn-off of $P_{f}$ are influenced by the difference in bath and lumen osmolalities, rather than the absolute osmolalities. For example, similar results were obtained for 100 mosM bath > lumen osmolalities when bath osmolality was 290 or 390 mosM. The $\left(T_{1 / 2}\right)_{\text {on }}$ and $\left(T_{1 / 2}\right)_{\text {off }}$ values correlated well with the magnitude and direction of the osmotic gradient.
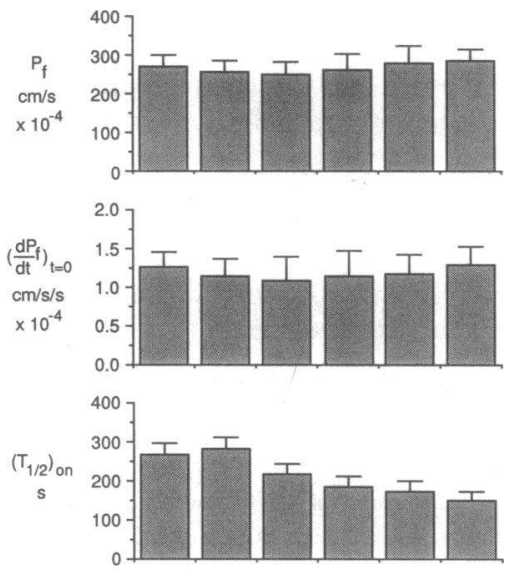

Figure 3. Effects of osmotic gradient size and direction on the vasopressin-dependent turnon and turn-off of water permeability. Experiments were carried out as in Fig. 1 for a series of different bath and lumen osmolalities. The measured quantities are steady-state $P_{f}$, the initial rate of turn-on of $P_{f}$ $\left(\mathrm{d} P_{\mathrm{f}} / \mathrm{d} t\right)_{t=0}$ after VP

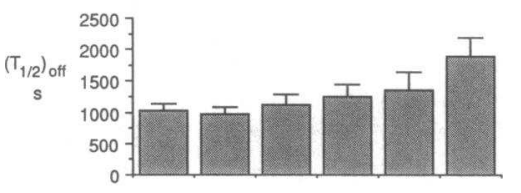
addition, $\left(T_{1 / 2}\right)_{\text {on }}$, and $\left(T_{1 / 2}\right)_{\text {of. }}$ Data are mean \pm SE for experiments performed on six to eight separate tubules for each set of bath and lumen osmolalities.

Based on freeze-fracture electron micrographs in vasopressin-responsive epithelia showing a strong correlation between $P_{f}$ and the appearance of apical membrane particle aggregates (3-5), it is assumed that the time course of $P_{f}$ increase is a measure of membrane exocytosis. To support the interpretation that the time course of $P_{f}$ decrease is a measure of membrane endocytosis, uptake of rhodamine-dextran, a fluid phase marker of endocytosis, was measured after vasopressin removal in the presence of bath $>$ lumen, lumen $>$ bath, and lumen $=$ bath osmolalities. The protocol given in the legend to Fig. 1 was used except that rhodamine-dextran was added to the lumen solution at the time of vasopressin removal (see Methods). After an additional $10 \mathrm{~min}$ of perfusion, rhodaminedextran was removed from the lumen solution for determination of cell fluorescence. Examination of tubule cells at high magnification $(\times 2,000)$ by Nipkow wheel confocal microscopy (Technical Instrument Co., San Francisco, CA) showed that virtually all rhodamine fluorescence was in small vesicles, similar to morphology reported previously (14). In paired experiments where the same tubule was used for uptake studies in the absence and presence of a transepithelial osmotic gradient, the bath $>$ lumen and lumen $>$ bath gradients gave $168 \pm 14 \%$ (SEM, $n=4)$ and $82 \pm 8 \%(n=4)$ (Fig. 4), respectively, of the rhodamine-dextran uptake measured in the absence of an osmotic gradient. Rhodamine-dextran uptake decreased by $>85 \%$ when temperature was $10^{\circ} \mathrm{C}$ to inhibit endocytosis. These results support the interpretation that the osmotic gradient direction and size influences rates of water channel endocytosis in CCT (see Discussion).

To examine the specificity of the osmotic gradient effect on endocytosis, experiments were carried in rabbit PST. Water transport and endocytosis in PST is hormone insensitive. Fig. 4 shows that neither bath-to-lumen nor lumen-to-bath osmotic gradients influenced the uptake of rhodamine-dextran. Rhodamine dextran uptake was inhibited by $>80 \%$ at $10^{\circ} \mathrm{C}$. The rate of transcellular water flow in PST in the presence of a 40-mosM osmotic gradient was considerably greater than that in CCT 


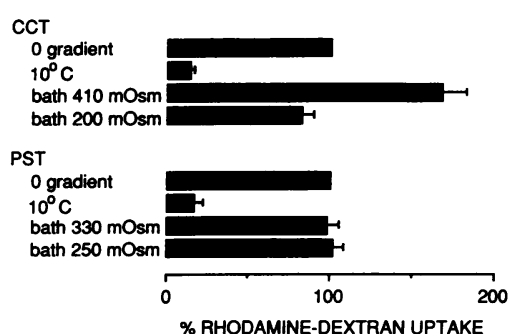

Figure 4. Endocytosis of rhodamine-dextran in CCT and PST. Luminal rhodamine-dextran uptake was measured after a 10-min labeling period under control conditions ( 290 mosM bath and luminal solutions) and under test conditions (see Methods).

Test conditions consisted of osmotic gradients or low temperature. In CCT studies, vasopressin was present in the bath. Data are the mean \pm SEM for three to five tubules.

because of the higher $P_{f}$ in PST $(0.2-0.5 \mathrm{~cm} / \mathrm{s}$, refs. 29,33$)$ than in CCT $(0.02-0.03 \mathrm{~cm} / \mathrm{s})$.

Although the magnitude and direction of transcellular volume flow in CCT provides a plausible explanation for the observed asymmetry in the kinetics of turn-on and turn-off of $P_{f}$, other potentially important mechanisms exist. There are signifcant changes in principal cell morphology induced by osmotic gradients $(30,34)$. Rectification in steady-state osmotic water flow has been reported when lumen osmolality was 125 or 490 mosM and bath osmolality was 290 mosM (35). It was concluded that the asymmetry was due to a dependence of the leakiness of tight junctions on osmotic gradient direction. To investigate this possibility in our experiments, the dependence of steady-state volume flow on osmotic gradient size and direction was measured in the absence and presence of vasopressin (Fig. 5). A linear dependence of $J_{v}$ on the log mean osmotic gradient size was observed without evidence of asymmetry. In the absence of vasopressin, $J_{v}$ measured from the fluorescence at the distal end of the tubule (see Methods) changed in $<2 \mathrm{~s}$ and remained constant for $>10 \mathrm{~min}$ in response to a change in bath osmolality. Thus, under our experimental conditions of constant lumen osmolality and relatively small osmotic gradients, there was no evidence of asymmetric paracellular water flow. In the presence of vasopressin, $J_{v}$ was also symmetric when measured within the first $10 \mathrm{~min}$ after a change in bath osmolality; at longer times in the sustained presence of vasopressin, small changes in $P_{f}$ have been observed in the presence of an osmotic gradient (23). It was also shown previously (see Fig. 4 of ref 12) that pre-steady-state phenomena were absent in the vasopressin-stimulated CCT in response to a change in bath osmolality. From the theoretical analysis of Schafer et al. (36), this finding indicates the absence of significant unstirred

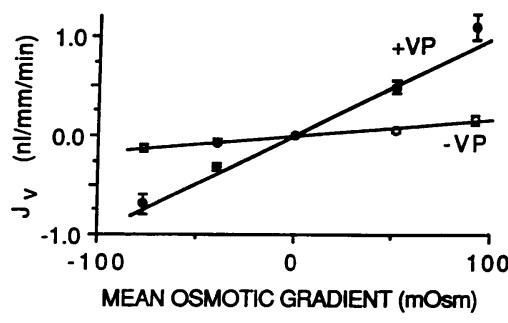

Figure 5. Dependence of $J_{v}$ on log mean osmotic gradient. $\mathrm{J}_{\mathrm{v}}$ $($ mean \pm SEM) for six tubules was determined in the presence and $a b$ sence of $250 \mu \mathrm{U} / \mathrm{ml}$ vasopressin as described in Methods. In each tubule, fluorescence measurements were made 1

min after setting bath osmolalities to the values: $210,250,290,350$, and 410 mosM. Linear fits are given. The range of $P_{f}$ values were $(234-277) \times 10^{-4} \mathrm{~cm} / \mathrm{s}(+\mathrm{VP})$, and $(10-15) \times 10^{-4} \mathrm{~cm} / \mathrm{s}(-\mathrm{VP})$.

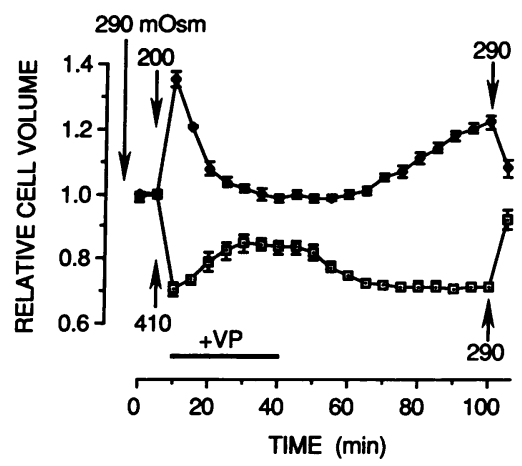

Figure 6. Time course of principal cell volume measured in eight tubules subject to a bathto-lumen osmotic gradient (bath 410 mosM) and seven tubules subject to a lumen-to-bath osmotic gradient (bath 200 mosM). Relative cell volume (mean \pm SEM) was measured by manual cell tracings from recorded images as described in Methods. Tubules were first perfused for 90 min (lumen and bath 290 mosM) in the absence of vasopressin. Where indicated, bath osmolalities were changed and $250 \mu \mathrm{U} / \mathrm{ml}$ vasopressin was present.

layer effects. These results show that the pre-steady-state, but not the steady-state, water permeability properties of cortical collecting tubule depend upon the magnitude and direction of the transepithelial osmotic gradient.

Another possible mechanism by which osmotic gradients could influence the kinetics of turn-on and turn-off of $P_{f}$ is by asymmetric changes in cell volume. To investigate this possibility, the time course of cell volume was measured directly in principal cells using established imaging techniques (Fig. 6) $(30,31)$. The experimental protocol was the same as that given in Fig. 1. With an increase in bath osmolality from 290 to 410 mosM, cell volume rapidly decreased to $72 \%$ of initial volume. Upon vasopressin addition and increasing apical membrane water permeability, the volume increased to $85 \%$ with a halftime of $10 \mathrm{~min}$; volume decreased again after vasopressin removal. Reciprocal changes were observed when bath osmolality was changed from 290 to 200 mosM. In both cases the time course of cell volume was quite similar to the time course of $P_{f}$ given in Figs. 1 and 2.

\section{Discussion}

The purpose of our study was to examine the effects of transcellular water flow on the vasopressin-dependent movement of subcellular vesicles containing water channels. The impetus for this study was the observations in toad urinary bladder that a serosal-to-mucosal osmotic gradient stimulated membrane retrieval $(20,22)$. Based on the toad bladder data and the collecting tubule data reported here, we proposed that water flow itself might provide a physical force to influence the targeted movement of membranes within a cell. In toad bladder, the presence of a serosal-to-mucosal osmotic gradient caused formation of an increased number of endosomes; at submaximal concentrations of vasopressin, the water permeability of individual endosomes decreased (7). It was suggested that water flow from lumen into the cell caused the premature detachment of apical membrane patches before they were fully packed with water channels.

A kinetic approach was required to examine the hypothesis that water flow modulates vesicle traffic. The isolated perfused cortical collecting tubule from rabbit was selected because transcellular water flow: $(a)$ is hormone-responsive and very rapid; (b) involves membrane cycling $(9,33)$; and $(c)$ is not limited by unstirred layer effects $(23,31)$. Similar studies could 
not be performed in isolated or cultured cells because steadystate transcellular water flow is required. We developed quantitative fluorescence microscopy techniques recently to record continuously the magnitude of transepithelial osmotic and diffusional water permeability based on measurement of the fluorescence of impermeant fluorophores perfused through the tubule lumen $(23,27)$. Using this new methodology, the data reported here show that osmotic gradients strongly influence the kinetics of turn-on and turn-off of water permeability in kidney collecting tubule.

Based on several lines of evidence, it is argued that it is transcellular volume movement that influences water channel exocytosis and endocytosis. The turn-on of water permeability after vasopressin addition, assumed to represent exocytosis of water channel-containing membranes, is enhanced by a lumen-to-bath gradient and inhibited by a bath-to-lumen gradient only after transcellular water flow has reached a threshold value (Figs. 2 and 3). The turn-off of water permeability after vasopressin removal, at a time when transcellular water flow is high, is enhanced without delay by a bath-to-lumen gradient and inhibited by a lumen-to-bath gradient. The effects of osmotic gradient size and direction on the turn-on and turn-off of water permeability were not dependent upon absolute osmolalities (Fig. 3). Parallel experiments in which the endocytic uptake of rhodamine-dextran was measured show a correlation between rates of endocytic retrieval and turn-off of water permeability in response to vasopressin removal. Importantly, it is the kinetics of the turn-on and turn-off of $P_{f}$, and not the steady-state values, which are influenced by the magnitude and direction of osmotic gradients.

Control studies were performed to evaluate a series of alternative explanations for the observation that the direction and magnitude of transcellular water flow influenced the kinetics of turn-on and turn-off of water permeability and the rates of endocytosis. Under some conditions it has been reported that rectification of vasopressin-independent transepithelial water movement exists because of asymmetry in paracellular permeability properties (35). We found that both vasopressin-independent and dependent water transport are symmetrical and not altered significantly for at least $10 \mathrm{~min}$ following a change in bath osmolality. Therefore our observed asymmetry cannot be due to paracellular transport or unstirred layer effects. Changes in cell volume and/or cell morphology in response to water flow in different directions might cause asymmetrical water transport properties $(30,31,34)$. We find, however, that the steady-state transepithelial $P_{f}$ of tubules in the presence or absence of vasopressin does not depend upon the magnitude or direction of water flow. Furthermore, the changes in cell volume paralleled the kinetics of turn-on and turn-off of $P_{f}$, suggesting that cell volume changes occur secondary to changes in apical membrane water permeability. It is unlikely that a small increase or decrease in cell volume had a direct effect on vesicle traffic because at the time of vasopressin removal, relative cell volume was near unity for experiments with bath $>$ lumen and lumen $>$ bath osmolalities. Furthermore, it was reported that cell swelling may cause a decreased rate of exocytosis (37), opposite to results reported here. Finally, rhodamine-dextran uptake experiments performed in proximal tubule, in which endocytosis is constitutive rather than vasopressin dependent, showed that the influence of water flow on vesicle traffic in collecting tubule is not a generalized, nonspecific phenomenon.
Our experiments provide evidence that, in kidney collecting tubule cells, transcellular volume flow modulates the vasopressin-dependent exocytic-endocytic cycling of intracellular membranes containing water channels. The data support the novel hypothesis that transcellular volume flow provides a physical driving mechanism to aid or oppose the targeted, hormonally directed movement of intracellular membranes. Physiologically, interstitial osmolality in renal cortex is always $\sim 300$ mosM, and the cortical collecting is responsible for the greatest quantity of solute-free water absorption in antidiuresis. Our data suggest that the osmolality of tubular fluid in the lumen of cortical collecting tubule is an important determinant of the kinetics of vasopressin action.

\section{Acknowledgments}

This work was supported by grants DK-35124, DK-39354, and HL42368 from the National Institutes of Health, and a grant from the National Cystic Fibrosis Foundation. Dr. Verkman is an established investigator of the American Heart Association.

\section{References}

1. Muller, J., W. A. Kachadorian, and V. A. DiScala. 1980. Evidence that ADH-stimulated intramembrane particle aggregates are transferred from cytoplasmic to luminal membranes in toad bladder epithelial cells. J. Cell Biol. 85:83-93.

2. Wade, J. B., D. L. Stetson, and S. A. Lewis. 1981. ADH action: evidence for a membrane shuttle hypothesis. Ann. NY Acad. Sci. 372:106-117.

3. Bourguet, J., J. Chevalier, and J. S. Hugon. 1976. Alterations in membraneassociated particle distribution during antidiruetic challenge in frog urinary bladder epithelium. Biophys. J. 16:627-639.

4. Harmanci, M. C., P. Stern, W. A. Kachadorian, H. Valtin, and V. A DiScala. 1980. Vasopressin and collecting duct intramembranous particle clusters: a dose-response relationship. Am. J. Physiol. 239:F560-F564.

5. Brown, D., A. Grosso, and R. C. DeSousa. 1983. Correlation between water flow and intramembrane particle aggregates in toad epidermis. Am. J. Physiol. 245:C334-C342.

6. Shi, L.-B., and A. S. Verkman. 1989. Very high water permeability in vasopressin-dependent endocytic vesicles in toad urinary bladder. J. Gen. Physiol. 94:1101-1115.

7. Shi, L.-B., D. Brown, and A. S. Verkman. 1990. Water, urea and proton transport properties of endosomes containing the vasopressin-sensitive water channel from toad bladder. J. Gen. Physiol. 95:941-960.

8. Shi, L.-B., Y.-X. Wang, and A. S. Verkman. 1990. Regulation of the formation and water permeability of endosomes from toad bladder granular cells. $J$. Gen. Physiol. 96:789-808.

9. Handler, J. S. 1988. Antidiuretic hormone moves membranes. Am. J. Physiol. 255:F375-F382.

10. Brown, D., and L. Orci. 1983. Vasopressin stimulates formation of coated pits in rat kidney collecting ducts. Nature (Lond.). 302:253-255.

11. Strange, K., M. C. Willingham, J. S. Handler, and H. W. Harris. 1988 Apical membrane endocytosis via coated pits is stimulated by removal of antidiuretic hormone from isolated, perfused rabbit cortical collecting tubule. $J$. Membr. Biol. 103:17-28.

12. Verkman, A. S., P. Weyer, D. Brown, and D. A. Ausiello. 1989. Functional water channels are present in clathrin coated vesicles from bovine kidney but not from brain. J. Biol. Chem. 264:20608-20613.

13. Verkman, A. S., W. Lencer, D. Brown, and D. A. Ausiello. 1988. Endosomes from kidney collecting tubule cells contain the vasopressin-sensitive water channel. Nature (Lond.). 333:268-269.

14. Lencer, W., D. Brown, D. A. Ausiello, and A. S. Verkman. 1990. Endocytosis of water channels in rat kidney: cell specificity and correlation with in vivo antidiuretic states. Am. J. Physiol. 259:920-932.

15. Lencer, W. I., A. S. Verkman, D. A. Ausiello, A. Arnaout, and D. Brown 1990. Endocytic vesicles from renal papilla which retrieve the vasopressin-sensitive water channel do not contain an $\mathrm{H}^{+}$ATPase. J. Cell Biol. 111:379-389.

16. Lencer, W. I., P. Weyer, A. S. Verkman, D. A. Ausiello, and D. Brown. 1990. FITC-dextran as a probe for endosome function and localization in kidney Am. J. Physiol. 258:C309-C317.

17. Rodman, J. S., L. Seidman, and M. G. Farquhar. 1986. The membrane 
composition of coated pits, microvilli, endosomes and lysosomes is distinctive in the rat kidney proximal tubule cell. J. Cell Biol. 102:77-87.

18. Ye, R., L.-B. Shi, W. Lencer, and A. S. Verkman. 1989. Functional colocalization of water channels and proton pumps on endocytic vesicles from proximal tubule. J. Gen. Physiol. 93:885-902.

19. Bae, H.-R., and A. S. Verkman. 1990. Protein kinase A regulates chloride conductance in endocytic vesicles from proximal tubule. Nature (Lond.). 348:637-639.

20. Gronowicz, G., S. K. Masur, and E. Holtzman. 1980. Quantitative analysis of exocytosis and endocytosis in the hydroosmotic response of toad bladder. $J$. Membr. Biol. 52:221-235.

21. Masur, S. K., S. Cooper, and M. S. Rubin. 1984. Effect of an osmotic gradient on antidiuretic hormone-induced endocytosis and hydroosmosis in the toad urinary bladder. Am. J. Physiol. 247:F370-F379.

22. Harris, H. W., Jr., J. B. Wade, and J. S. Handler. 1986. Transepithelial water flow regulates apical membrane retrieval in antidiuretic hormone-stimulated toad urinary bladder. J. Clin. Invest. 78:703-712.

23. Kuwahara, M., C. A. Berry, and A. S. Verkman. 1988. Rapid development of vasopressin-induced hydroosmosis in kidney collecting tubules measured by a new fluorescence technique. Biophys. J. 54:595-602.

24. Kuwahara, M., and A. S. Verkman. 1989. Pre-steady-state analysis of the regulation of water permeability in the kidney collecting tubule. J. Membr. Biol. 110:57-65.

25. Levine, S. D., and W. A. Kachadorian. 1981. Barriers to water flow in vasopressin-treated toad urinary bladder. J. Membr. Biol. 61:135-139.

26. Burg, M., J. Grantham, M. Abramow, and J. Orloff. 1966. Preparation and study of fragments of single rabbit nephrons. Am. J. Physiol. 210:1293-1298.

27. Kuwahara, M., and A. S. Verkman. 1988. Direct fluorescence measure- ment of diffusional water permeability in the vasopressin-sensitive kidney collecting tubule. Biophys. J. 54:587-593.

28. Al-Zahid, B., J. A. Schafer, S. L. Troutman, and T. E. Andreoli. 1977. Effect of antidiuretic hormone on water and solute permeation, and the activation energies for these processes in mammalian cortical collecting tubules. $J$. Membr. Biol. 31:103-129.

29. Berry, C. A., and A. S. Verkman. 1988. Osmotic gradient dependence of water permeability in the rabbit proximal convoluted tubule. J. Membr. Biol. 105:33-43.

30. Kirk, K. L., J. A. Schafer, and D. B. DiBona. 1984. Quantitative analysis of the structural events associated with antidiuretic hormone-induced volume reabsorption in the rabbit cortical collecting tubule. J. Membr. Biol. 79:65-74.

31. Strange, K., and K. R. Spring. 1987. Cell membrane water permeability of rabbit cortical collecting duct. J. Membr. Biol. 96:27-43.

32. Kirk, K. L. 1988. Origin of ADH-induced vacuoles in rabbit cortical collecting tubule. Am. J. Physiol. 254:F719-F733.

33. Verkman, A.S. 1989. Mechanisms and regulation of water permeability in renal epithelia. Am. J. Physiol. 257:C837-C850.

34. DiBona, D. R. 1983. Cytoplasmic involvement in ADH-mediated osmosis across toad urinary bladder. Am. J. Physiol. 245:C297-C307.

35. Schafer, J. A., S. L. Troutman, and T. E. Andreoli. 1974. Osmosis in cortical collecting tubules: ADH-independent osmotic flow rectification. J. Gen. Physiol. 64:228-240.

36. Schafer, J. A., C. S. Patlak, and T. E. Andreoli. 1974. Osmosis in cortical collecting tubules: a theoretical and experimental analysis of the osmotic transient phenomenon. J. Gen. Physiol. 64:201-227.

37. Willingham, M. C., and I. Pastan. 1984. Endocytosis and exocytosis: current concepts of vesicle traffic in animal cells. Int. Rev. Cytol. 92:51-92. 